\title{
A heurística de Boltzmann e a emergência do programa mecânico-estatístico
}

(Boltzman's heuristics and the emergence of the mechanical-statistical program)

\author{
Cássio C. Laranjeiras ${ }^{1}$ e José R.N. Chiappin² \\ ${ }^{1}$ Instituto de Fisica, Universidade de Brasília, Brasilia, DF, Brasil \\ ${ }^{2}$ Departamento de Filosofia, Universidade de São Paulo, São Paulo, SP, Brasil
}

\begin{abstract}
O objetivo deste trabalho é investigar a construção de uma abordagem estatística como recurso heurístico de compreensão das relações entre mecânica e termodinâmica no interior do programa de pesquisa de Ludwig Boltzmann (1844-1906). Neste contexto, a função de distribuição das velocidades moleculares de J.C. Maxwell (1831-79), acerca da qual Boltzmann fará diferentes leituras, desempenhará um papel central na medida em que é a partir dela que novos instrumentos heurísticos surgirão, tais como os conceitos de "ensemble estatístico" e "probabilidade de estado", que representam um esforço conceitual no sentido de associar aos estados do fenômeno sob análise a probabilidade da sua realização.
\end{abstract}

Palavras-chave: mecânica estatística, Ludwig Boltzmann, heurística.

The purpose of this paper is to investigate the construction of a statistical approach as a heuristic tool to understand the relations between mechanics and thermodynamics inside Ludwig Boltzmann's (1844-1906) research program. In this context, J.C Maxwell's (1831-79) distribution function of molecular velocities, which Boltzmann interpreted in different ways, will have a central role for being the source of new heuristic tools, such as the "statistical ensemble" and the "probability of state". These represent a conceptual effort as they associate the probability of its realization to the state of the phenomenon under analysis.

Keywords: statistical mechanics, Ludwig Boltzmann, heuristics.

\section{Introdução}

O programa de pesquisa de Ludwig Boltzmann (18441906) se insere no contexto do programa mecanicista do século XIX comprometido em ampliar e colocar em operação os recursos da mecânica, tomada por ele como uma representação adequada e unificadora dos fenômenos da natureza, com vistas à resolução de problemas no âmbito da física teóric ${ }^{2}$. Assim é que, em seu esforço por compreender e explicar, sob uma perspectiva microscópica, as propriedades observáveis da matéria, Boltzmann se viu diante do desafio de definição e explicação do equilíbrio termodinâmico. Do ponto de vista macroscópico, os processos termodinâmicos são extremamente estáveis e regulares, o que era de difícil reconciliação com o fato de que os sistemas mecânicos são compostos por uma enorme quantidade de partículas que se movem com um movimento bastante irregular. Além do mais, os sistemas termodinâmicos não são unicamente estáveis, mas também irreversíveis, embora a reversibilidade seja to- talmente apropriada ao comportamento individual das partículas que compõem esses sistemas. Ao tentar explicar a irreversibilidade, Boltzmann foi levado à investigação das propriedades moleculares dos estados termodinâmicos e a desenvolver um tratamento geral do equilíbrio térmico, ultrapassando assim, as abordagens correntes em teoria cinética dos gases, desenvolvidas por R. Clausius (1822-88) e J.C. Maxwell (1831-79).

A utilização das ferramentas da mecânica analítica, guiadas por sua perspectiva atomista de entendimento da estrutura da matéria, lhe possibilitou o tratamento matemático do movimento microscópico e a conseqüente construção de uma abordagem estatística como recurso heurístico de compreensão das relações entre mecânica e termodinâmica no interior do seu programa de pesquisa. Neste contexto, a função de distribuição das velocidades moleculares de Maxwell, acerca da qual Boltzmann fará diferentes leituras, desempenhará um papel central na medida em que é a partir dela que novos instrumentos heurísticos surgirão, tais como os conceitos de "ensemble estatístico" e "probabilidade

\footnotetext{
${ }^{1}$ E-mail: cassio@unb.br.

Copyright by the Sociedade Brasileira de Física. Printed in Brazil.

${ }^{2}$ Uma discussão mais ampla do Programa de Pesquisa de Boltzmann para a Mecânica Estatística pode ser encontrada em [1].
} 
de estado", que representam um esforço conceitual no sentido de associar aos estados do fenômeno sob análise a probabilidade da sua realização. Neste sentido, identificamos em Boltzmann não somente a construção daquilo que, numa linguagem estatística se conhece como "espaço amostral" 3, o espaço dos eventos, o conjunto de todos os possíveis resultados de uma determinada experiência, mas também o esforço em atribuir probabilidades a esse espaço, conduzindo-nos à noção modernamente construída de "espaços de probabilidades" "4] É como elo de ligação entre os dois que se pode identificar a noção de "ensemble" no programa de Boltzmann.

\section{A transição de uma abordagem ciné- tica para uma abordagem estatística}

O marco referencial da construção do programa de pesquisa de Boltzmann foi a publicação do artigo "Sobre o significado mecânico da $2^{\mathrm{a}}$ lei da termodinâmica" [2], onde ele buscou, através de uma abordagem cinética, compreender a irreversibilidade termodinâmica no quadro referencial da mecânica. Atacar o problema da irreversibilidade significava conciliar o caráter irregular, reversível do movimento dos elementos constituintes de um dado sistema, com o caráter regular, irreversível, apresentado por esses mesmos sistemas quando vistos de um ponto de vista macroscópico. Naquele momento, os recursos de que Boltzmann dispunha eram, além dos conceitos e leis da mecânica, dos conceitos e princípios da termodinâmica estabelecidos por Clausius (182288), àqueles gestados no interior do Programa CinéticoMolecular, cujo núcleo central era a idéia de que o comportamento e a natureza das substâncias resultam do movimento de uma enorme quantidade de elementos que, em última instância, obedecem às leis da mecânica. Do ponto de vista heurístico a estratégia de Boltzmann neste momento era remeter os fenômenos macroscópicos à estrutura da matéria e ao movimento individual dos seus elementos constituintes (átomos), através da aplicação dos princípios da mecânica ao comportamento desses elementos. Desta forma ele se propôs enfrentar o problema da irreversibilidade, tendo conseguido estabelecer uma analogia mecânica para a entropia através da prova de um teorema reconhecido como a forma generalizada do princípio da mínima açã $c^{5}$. Apesar disso, a explicação para o seu aumento irreversível continuou em aberto visto que em sua caracterização mecânica do estado de equilíbrio, Boltzmann se restringiu a sistemas estritamente periódicos e, portanto, reversíveis mecanicamente.

Foi no contato com a "função de distribuição das velocidades moleculares" de Maxwell (1831-79) que Boltzmann, através de diferentes leituras da mesma, encontrou novos elementos heurísticos para seus desenvolvimentos posteriores.

A função de distribuição de velocidades foi inicialmente derivada por Maxwell para o caso particular de um gás monoatômico em equilíbrio na ausência de forças externas e posteriormente generalizada pelo próprio Boltzmann numa série de $\operatorname{artigos}(3,5,6$, 6 . e [4]) dedicados ao estudo do equilíbrio térmico. Neles, Boltzmann generaliza a função de distribuição e estende o teorema de eqüipartição de energia para o caso de moléculas poliatômicas sob a ação de um potencial.

Diferente de 1866, quando o estado termodinâmico de um corpo foi definido microscopicamente por ele através do movimento de um único átomo, cuja validade foi estendida para todos os demais (abordagem cinética), a partir da função de distribuição de velocidades moleculares de Maxwell, Boltzmann vai falar do estado termodinâmico de um corpo em função dos limites das coordenadas dos átomos, estando estes limites definidos em termos de probabilidades ([4], 6]).

Portanto, o contato de Boltzmann com os trabalhos de Maxwell marca uma nova etapa no desenvolvimento do seu programa de pesquisa, que será marcada basicamente pelo uso de dois procedimentos:

1. Utilização da "função de distribuição" em substituição a um conjunto completo de variáveis moleculares

2. Substituição de argumentos de natureza cinética, ligados à descrição temporal do movimento irregular das partículas, por argumentos de natureza probabilística, que vão estabelecer as relações entre a evolução do sistema no tempo e os limites do movimento das partículas no espaço.

A partir desta nova abordagem começa a se delinear no programa de Boltzmann um novo método de tratamento do equilíbrio termodinâmico caracterizado, de um lado pela criação do "ensemble estatístico" e, por conseqüência, pela adoção de um novo espaço, que não é mais o espaço $\mu$ das partículas individuais, mas o espaço de todo o gás, o espaço $\Gamma$, denominado de "espaço de fase", espaço de todos os estados acessíveis ao sistema estudado; e de outro lado pela substituição da média temporal por uma média espacial, tomada sobre todo ensemble estatístico, na representação de uma

\footnotetext{
${ }^{3} \mathrm{Na}$ mecânica estatística ele corresponde ao conjunto de todos os estados microscópicos (microestados) acessíveis ao sistema, o espaço no interior do qual os estados estarão distribuídos com suas respectivas probabilidades.

${ }^{4}$ Do ponto de vista moderno um "espaço de probabilidades" é formado por um trio $(\Omega, \Lambda, P)$, onde $\Omega$ é um conjunto não vazio representando o espaço amostral. $\Lambda$ é um subconjunto de $\Omega$ (uma álgebra de eventos) e $P$ é uma probabilidade em $\Lambda$.

${ }^{5}$ Foi a partir da expressão:

$$
S=\iint \frac{\delta Q}{T} d k=2 \Sigma \log \int_{t_{1}}^{t_{2}} \frac{m c^{2}}{2} d t+C
$$

que Boltzmann estabeleceu formalmente as relações entre a quantidade de calor fornecida aos corpos e a variação do movimento de cada átomo no espaço, através da igualdade entre a variação da ação e a variação da energia cinética de cada átomo.
} 
dada quantidade física macroscópica (variável termodinâmica).

Precisemos um pouco melhor este nível heurístico no pensamento de Boltzmann, construído ao longo do ano de 1871, em cuja raiz se encontra uma importante hipótese que os Ehrenfests posteriormente apelidaram de "hipótese ergódica" ([6, p. 270], [12, p. 21]) e que marca de uma maneira bastante peculiar a transição, no programa de Boltzmann, de uma abordagem cinética para uma abordagem estatística.

Para calcularmos a média temporal de uma dada grandeza A no laboratório, normalmente tomamos a média dos seus valores num tempo muito grande $t$. Assim, podemos escrever

$$
<A>_{l a b}=\lim _{\tau \rightarrow \infty} \frac{1}{\tau} \int_{0}^{\tau} A(t) d t .
$$

Por outro lado pode-se imaginar um conjunto de sistemas distribuídos no espaço de fase ("ensemble estatístico") de tal forma que a densidade destes sistemas seja dada por $\rho(q, p)$. O valor médio da grandeza A no ensemble é então dado por

$$
<A>_{\text {ensemble }}=\frac{\int A(q, p) \rho(q, p) d q d p}{\int \rho(q, p) d q d p} .
$$

A hipótese de Boltzmann consistiu em supor que os valores médios definidos pelas Eqs. (2) e (3) são idênticos, e igual ao valor termodinâmico de A. Isto significa assumir que a média de uma função no tempo, obtida seguindo os pontos da sua trajetória, seria tomada sobre todos os pontos e, portanto, se igualaria à média de fase.

É na base desta suposição que vamos encontrar aquilo que os Ehrenfests apresentaram em 1911 como sendo a "justificativa de Boltzmann-Maxwell" [12, p. 21], que ficou conhecida como "hipótese ergódica", ou seja, a idéia de que a trajetória de fase de um sistema dinâmico (único) é tal que esta passa por todos os pontos compatíveis com sua energia total 6 .

$\mathrm{Na}$ visão de Von Plato [18, admitir esta idéia significa adotar uma leitura pouco atenta do trabalho de Boltzmann, para quem a idéia de uma trajetória única preenchendo o conjunto do espaço dos estados estava bastante distante do seu horizonte. De fato, Boltzmann admite a possibilidade de diferentes trajetórias (as figuras de Lissajous são o exemplo de movimento que ele utiliza), formulando a ergodicidade como a condição de existência de unicamente uma invariante do movimento: a energia total. Portanto, na impossibilidade de atribuir comportamento ergódico a um sistema único, o que significava admitir como possível a dependência teórica às condições iniciais, Boltzmann vai utilizar o que posteriormente ele caracterizou como sendo um truque ("Kunstgriff"), "a ficção de sistemas independentes infinitamente congruentes" [8, p.123], "Inbergriff von
Systeme" ("a mais alta representação do sistema"), os chamados "ensembles", como ficaram conhecidos após Gibbs. Para o caso específico aqui considerado, aqueles onde todos os sistemas têm a mesma energia, Boltzmann utilizou a expressão "Ergoden"7 ("ensemble microcanônico" de Gibbs). Desta forma, os "ensembles" são introduzidos como recurso heurístico na solução do problema de calcular as propriedades macroscópicas dos gases independentemente de sua evolução microscópica.

Posteriormente, o "método de ensemble" se tornou um instrumento fundamental para a física estatística através do trabalho de Willard Gibbs (1839-1903), que cita Boltzmann em seu prefácio de Principios Elementares de Mecânica Estatística [15, p. viii] como o pioneiro no uso desse tipo de representação.

Para justificar isto, Boltzmann considerou que durante a evolução do sistema, o tempo $\Delta t$ gasto em um dado elemento de volume $\Delta V$ do espaço de fase (discreto) é proporcional a este elemento de volume, ou seja,

$$
\lim _{t \rightarrow \infty} \frac{\Delta t}{t}=\frac{\Delta V}{V}
$$

onde $V$ é o volume total da região considerada.

Suponha que um dado sistema $S$ se encontre, durante um período de tempo suficientemente longo, $\tau$, no estado $S_{i}$ por um período de tempo $\tau_{i}$. Da mesma forma que nós podemos definir a freqüência relativa de um dado evento, pode-se definir a proporção relativa de tempo no qual o gás permanece naquele estado $\left(\frac{\tau_{i}}{\tau}\right)$. Esta foi a estratégia de Boltzmann ao identificar esta fração de tempo $\left(\frac{\tau_{i}}{\tau}\right)$ com a "probabilidade de estado".

Pode-se, portanto, compreender este período do trabalho de Boltzmann (1868-1871) como marcando o início da construção daquilo que, numa linguagem estatística, se conhece como "Espaço Amostral", o espaço dos eventos, que na perspectiva da mecânica estatística corresponde ao conjunto de todos os estados microscópicos (microestados) acessíveis ao sistema, o espaço no interior do qual os estados estarão distribuídos com suas respectivas probabilidades. Neste sentido, podemos visualizar no horizonte do programa Boltzmanniano para a mecânica estatística, cujo desdobramento reconhecemos no trabalho de Gibbs, o uso de dois tipos de "espaços amostrais", a saber:

Espaço Amostral I: aquele onde os estados estão distribuídos com igual probabilidade e que são representados pelos chamados "Ergoden" de Boltzmann("ensemble microcanônico" de Gibbs.)

Espaço Amostral II: aquele onde os estados se distribuem segundo diferentes pesos probabilísticos, dados pela distribuição de Maxwell-Boltzmann e represen-

\footnotetext{
${ }^{6}$ Esta posição tem sido motivo de controvérsias com importantes contribuições da parte de Brush [10, von Plato [18] e 13.

${ }^{7} \mathrm{~A}$ expressão foi introduzida explicitamente pela primeira vez por Boltzmann em 1884 [7].
} 
tados pelo que Boltzmann chamou de "Holode" ("ensemble canônico" de Gibbs.)

Os Ergoden de Boltzmann são caracterizados pela condição restritiva de que todos os sistemas pertencentes ao ensemble tenham a mesma energia. Utilizando este tipo de ensemble como método de análise para situações de equilíbrio, Boltzmann procurou resolver o problema da equipartição da energia entre os vários graus de liberdade do sistema, um resultado bem estabelecido por Clausius para o caso de um gás livre e por ele próprio para o caso de partículas interagentes. Mas a percepção de Boltzmann transcendeu ao caso particular de Ergoden. Numa carta dirigida a Helmholtz, que data de 27 de dezembro de 1844, ele reconhece a necessidade de ampliar o seu conceito de Ergoden (ensemble microcanônico) com o objetivo de encontrar uma classe apropriada de sistemas mecânicos análogos ao comportamento dos sistemas termodinâmicos.

Em seu artigo de 1885 [8, utilizando o que ele chama de truque ("Kunstgriff"), a ficção de sistemas independentes infinitamente congruentes, apoiado em resultados obtidos em alguns de seus artigos anteriores [4, 5, 6, Boltzmann vai estabelecer as propriedades essenciais de um ensemble, um novo método de análise alternativo ao método de evolução temporal que marcou o seu artigo de 1872, mostrando que os ensembles podem representar um sistema real em equilíbrio e que, portanto, podem funcionar como um modelo mecânico para a termodinâmica. Em contraposição ao método de evolução temporal, que utilizava como recurso heurístico um espaço de 6 dimensões, o espaço $\mu(\mathbf{x}, \mathbf{v}, t)$ (espaço da partícula), Boltzmann põe em operação um novo espaço de características mais amplas, que ao invés de olhar unicamente uma partícula, abrange agora o gás como um todo, se mostrando, portanto, mais eficiente na resolução de problemas no interior de sua teoria dos gases. Inaugura-se assim um novo recurso heurístico, o espaço $\Gamma$ (espaço do gás), um espaço de $6 n$ dimensões, sendo $n$ o número total de partículas no gás, onde se pode representar $H(q, p, t)$.

Ao longo do seu artigo, no contexto de análise deste novo método, o método de ensembles, Boltzmann introduz a noção de distribuição de probabilidade estacionária $^{9}$ sobre o espaço de fase de $N$ partículas interagentes encerradas em um recipiente de volume $V$, caracterizando um conjunto de tais probabilidades de um "Monode" (ensemble estacionário numa linguagem moderna), uma generalização do conceito de sistemas monocíclicos de Helmholtz.

Num primeiro momento Boltzmann utiliza a expressão "Monode" para se referir a um único sistema. Outras vezes, como bem observa Giovanni Gallavotti [13. p. 1572], implícita ou explicitamente, a mesma ex- pressão é usada para um conjunto de distribuições estacionárias. Com vistas a simplificar nossa discussão tomaremos a liberdade, a exemplo de Gallavotti, de usar a expressão "Monode" para representar um conjunto de distribuições estacionárias, denominando cada um dos sistemas individuais de "elemento de um monode" $(\mu)$.

Trabalhando com a hipótese de que entre as possíveis distribuições estacionárias, algumas podem ser interpretadas como estados de equilíbrio macroscópico, ele introduz a noção de "Orthode (ensemble de equilíbrio numa linguagem moderna)", cujas "equações são completamente análogas àquelas da teoria mecânica do calor" [8, p. 130].

Indo mais além, ele admite a existência de no mínimo dois tipos de "Orthode", parametrizados diferentemente, mas ambos capazes de representar um sistema em equilíbrio térmico, a saber:

"Holode" (ensemble canônico de Gibbs)

Parâmetros: $\beta$ (temperatura), $N$ (n. de partículas). $\mu_{\beta, N}$ : Elemento de um ensemble canônico

$$
\mu_{\beta, N}(d p d q)=\frac{d p_{1} \ldots d p_{n} d q_{1} \ldots d q_{n}}{\text { const. }} e^{-\beta(K+\phi)}
$$

"Ergode" (ensemble microcanônico de Gibbs) Parâmetros: $U$ (energia), $N$ (n. de partículas). $\mu_{U, N}$ : Elemento de um ensemble microcanônico

$$
\begin{aligned}
& \mu_{U, N}(d p d q)=\frac{d p_{1} \ldots d p_{n} d q_{1} \ldots d q_{n}}{\text { const. }} \\
& \delta(K(p)+\phi(q)-U) .
\end{aligned}
$$

Desta forma Boltzmann estabeleceu os ensembles canônicos e microcanônicos como representantes legítimos do equilíbrio térmico de um dado sistema, provendo desta forma modelos mecânicos para a termodinâmica.

Neste sentido, a tarefa da Mecânica Estatística nascente neste momento será a de construir estratégias para se atribuir probabilidades ao espaço dos estados.

Portanto, a emergência do Programa MecânicoEstatístico pode ser caracterizada, no programa de Boltzmann, a partir da transição de uma abordagem cinética para uma abordagem estatística, tendo como instrumento heurístico de mediação a função de distribuição de velocidades de Maxwell. Numa linguagem estatística podemos dizer que na abordagem cinética Boltzmann atribuía à amostra (molécula) a propriedade média da população (gás). Na abordagem estatística a função de distribuição vai formar a base das argumentações que lhe permitirão essa extrapolação.

Para que possamos ter uma melhor clareza desse processo de transição, relaciono em seguida os elementos característicos de cada uma dessas abordagens:

\footnotetext{
${ }^{8}$ No cap. 10, onde a construção de uma "teoria de ensemble" no programa de Boltzmann é discutida, esta noção aqui apenas citada, será apresentada.

${ }^{9}$ A noção de estacionaridade é devida a Maxwell em [Maxwell, 1879, p. 715.$]$
} 


\section{Abordagem cinética}

i) Uso de argumentos de natureza cinética ligados a descrição temporal do movimento irregular das partículas que compõem o sistema sob investigação.

ii) A descrição matemática do estado $S_{i}$ de um dado sistema $\mathrm{S}$ por um conjunto de pontos $\left(r_{1}, r_{2}, \ldots r_{n}\right)$ em um espaço euclidiano de 6 dimensões $\left(\right.$ Espaço- $\mu$ ), onde $r_{i}$ representa as três coordenadas de posição e momento da partícula $i$.

iii) Uso de Médias Temporais no cálculo das grandezas termodinâmicas

\section{Abordagem estatística}

i) Uso de argumentos de natureza probabilística.

ii) A descrição matemática do estado $S_{i}$ de um dado sistema $S$ por um único ponto em um espaço de dimensão $6 N$, onde $N$ é o número total de partículas no sistema (Espaço- $\Gamma)$. Para cada $r_{1}, r_{2}, \ldots r_{n}$ no espaço $\mu$ corresponde um ponto no espaço $\Gamma$.

iii) A noção de "ensemble estatístico".

iv) Uso de médias espaciais calculadas no "ensemble estatístico" para o cálculo das grandezas termodinâmicas.

As seções seguintes procuram abordar em maiores detalhes a construção dessa transição para uma abordagem mecânico-estatística.

\section{A distribuição de Maxwell-Boltz- mann: $\mathrm{O}$ método cinético e o método combinatorial}

Em 1868, com Estudos Sobre o Equilíbrio das Forças Vivas [Energia Cinética] entre Pontos Materiais em Movimento (3), Boltzmann estendeu a distribuição de equilíbrio para as velocidades moleculares de Maxwell num gás monoatômico, tratando o caso quando um campo de forças externo, como por exemplo o campo gravitacional, está presente (Distribuição de MaxwellBoltzmann). Nesta oportunidade ele nos apresentou dois diferentes métodos para alcançar este objetivo: o método cinético e o método combinatorial.

\subsection{O método cinético}

Neste método, Boltzmann parte das mesmas considerações cinéticas de Maxwell em On the Dynamical Theory of Gases [17, só que desta vez considerando o caso quando um campo de forças externo, como por exemplo o campo gravitacional está presente. Estas considerações são as seguintes:
1. O estado de equilíbrio é resultado da colisão entre as moléculas (argumento cinético).

2. As velocidades das moléculas em colisão são estatisticamente independentes, o que significa desconsiderar qualquer correlação entre as moléculas que colidem. Esta afirmação recebeu por parte de Boltzmann a denominação de "Stosszahlansatz" ("caos molecular").

\section{Conservação da energia.}

Seu ponto de partida, explicitado logo na introdução do artigo de 1868, é um questionamento acerca do comportamento das moléculas, caracterizadas como pontos materiais, quando deixadas sozinhas por um longo tempo.

Todos os princípios da mecânica analítica até agora desenvolvidos limitam-se à passagem de um sistema de pontos materiais de um certo estado a outro, a despeito das leis de mudança daquela situação e da velocidade de tais pontos materiais eles são deixados a si mesmos por um longo tempo em movimento, com exceção da teoria dos gases ideais com respeito a teoremas ainda quase nada conhecidos. Da mesma forma os teoremas da teoria mecânica do calor, que se referem a tais movimentos, até agora permanecem incoerentes em seus fundamentos analíticos. Eu quero em seguida dar vários exemplos e finalmente calcular um teorema geral para a probabilidade das diferentes posições e velocidades dos pontos materiais em movimento. [3, p. 49]

Insatisfeito com a derivação da função de distribuição feita por Maxwell, que ele considerava difícil de ser entendida devido a sua breve apresentação, Boltzmann dedica a primeira seção (Reflexões Sobre uma Infinidade de Pontos Materiais) do seu artigo, para preencher algumas lacunas e ilustrar com exemplos concretos alguns aspectos que, segundo ele, Maxwell tinha encoberto, tal como a natureza da função de distribuição.

É aqui que nós encontramos pela primeira vez a sua primeira definição da noção de probabilidade, concebida como média temporal.

Se $\varphi(c) d c$ é a soma do tempo durante o qual a velocidade de qualquer esfera, no curso de um tempo muito grande, jaz entre $c$ e $c+d c$ dividido por este tempo muito grande, portanto a probabilidade de $\mathrm{c}$ estar entre $c$ e $c+d c$ e $N$ o número de esferas, então

$$
N \varphi(c) d c
$$

é o número de esferas por unidade de superfície cujas velocidades estão entre $c$ e $c+d c$. [3, p. 50] 
A probabilidade é aqui identificada com a fração de um intervalo de tempo suficientemente longo, durante o qual a velocidade de uma molécula específica tem valores dentro de um certo elemento de volume no espaço das velocidades. Posteriormente, como veremos mais adiante em seu artigo de 1871, as probabilidades vão aparecer de uma forma bem mais explícita, vinculadas à noção de estado de um sistema, que será definido em função dos limites das coodenadas dos átomos.

A noção de probabilidade concebida como média temporal encontra sua origem já no artigo de 1866 , quando Boltzmann trata a temperatura como função da energia cinética média de cada molécula no tempo [2, p. 14]. Na ocasião, mesmo sem empregar a palavra probabilidade Boltzmann fez uso de noções probabilísticas em seus raciocínios.

Analisando o processo de colisão de duas moléculas, agora sob a ação de um potencial, ele se coloca o seguinte problema:

Ao longo de uma linha OX move-se uma esfera elástica com massa $M$, que é atraída para $\mathrm{O}$ por uma força que é uma função unicamente da distância do seu centro a $\mathrm{O}$. Ela está sendo continuamente bombardeada por esferas elásticas de massa $m$ com várias velocidades em intervalos de tempo irregulares, tal que se nós olhamos as esferas que bombardeiam distante de $\mathrm{O}$ sobre a linha OX, então o número de esferas com velocidades entre $c$ e $c+d c$ encontradas em médias sobre a unidade de comprimento é uma função definida de $c, N \phi(c) d c$. O potencial da força com que $M$ é atraída para O é $\chi(x)$, então, contanto que o movimento não seja perturbado por colisões, nós temos:

$$
\text { (9) } \frac{M C^{2}}{2}=\chi(x)+A \text {, }
$$

onde $C$ é a velocidade da esfera $M$ e $x$ a distância do seu centro a partir de O. A natureza da colisão é completamente determinada por três quantidades $x, A$ e $c$. O tempo durante o qual, no curso da unidade de tempo a constante $A$ da Eq. (9) jaz entre os valores $A$ e $A+d A$ será $\phi(A) d A$. [3, p. 61]

O problema aqui colocado era o de encontrar a fração de tempo durante a qual $A$ está entre $A$ e $A+d A$ e $x$ entre $x$ e $x+d x$. Boltzmann concluiu que esta fração de tempo é proporcional a $e^{-h A} d A d x$, ou seja:

$$
\phi(A) \alpha(\text { const. }) e^{h\left[\chi(x)-\frac{M C^{2}}{2}\right]} .
$$

A condição de conservação da energia cinética na derivação de Maxwell ${ }^{10}$ é substituída pela condição de conservação da energia total (cinética mais potencial) na derivação de Boltzmann, a saber:

$$
\frac{1}{2} m c^{2}+\chi\left(x_{1}\right)+\frac{1}{2} M C^{2}=\frac{1}{2} m c^{\prime 2}+\chi\left(x_{2}^{\prime}\right)+\frac{1}{2} M C^{\prime 2} .
$$

Com o estabelecimento dessa nova condição (a existência de um campo de forças externo) foi possível escrever a seguinte expressão para a função de distribuição:

$$
f(c)=B e^{-h\left[\frac{1}{2} m c^{2}+\chi(x)\right]}
$$

onde $B$ e $h$ são constantes. A constante $h$ está relacionada à temperatura absoluta e é modernamente equivalente a $\frac{1}{K T}$, sendo $K$ a constante de Boltzmann 11 .

A função definida pela Eq. (10) é atualmente conhecida como distribuição de Maxwell-Boltzmann, e nos dá a probabilidade de que uma molécula seja encontrada em um lugar com uma dada energia $E=\frac{1}{2} m c^{2}+\chi(x)$. O fator $e^{-h E}$, atualmente conhecido como "fator de Boltzmann", é agora amplamente utilizado em física molecular, visto que $\chi$ pode ser a função energia potencial para todas as forças atuando sobre a molécula, incluindo aquelas de outras moléculas. Combinado com a lei de distribuição de Maxwell, o "fator de Boltzmann $\left(e^{-h E}\right)$ " nos dá a probabilidade de qualquer estado molecular com energia total $E=\sum \frac{1}{2} m c^{2}+\sum \chi$.

Guttmann [19, p. 18] observa que em seus últimos escritos, Boltzmann se tornou cada vez mais consciente do quão problemática era a introdução do termo "Stosszahlansatz", ou seja, a afirmação de "caos molecular". Isso se depreende da cuidadosa exposição que Boltzmann faz das idéias de Maxwell em sua obra "Vorlesungen über Gastheorie" [9]. Esta sempre foi uma preocupação de Boltzmann, a ponto de, na segunda seção do seu artigo ( $O$ Equilíbrio das Forças Vivas [Energia Cinétical entre um Número Finito de Pontos Materiais), ele buscar um caminho diferente (ainda utilizando o método cinético) para a derivação da distribuição de Maxwell.

Num plano muito grande fechado por todos os lados encontram-se $n$ pontos materiais de massas $m_{1}, m_{2}, \ldots, m_{n}$ e velocidades $c_{1}, c_{2}, \ldots, c_{n}$, respectivamente, e entre

${ }^{10}$ Esta condição é representada pela expressão:

$$
\frac{1}{2} m_{1} v_{1}^{2}+\frac{1}{2} m_{2} v_{2}^{2}=\frac{1}{2} m_{1} v_{1^{\prime}}^{2}+\frac{1}{2} m_{2} v_{2^{\prime}}^{2} .
$$

${ }^{11}$ É importante lembrar aqui que Boltzmann não deduziu o valor de $K$, que foi posteriormente introduzido por Planck em conexão com sua lei da radiação (1900). 
os quais atuam determinadas forças... A probabilidade de que a velocidade de um ponto esteja entre determinados limites e ao mesmo tempo a velocidade de um outro se encontre entre outros limites, não será de modo algum produto de cada uma das probabilidades; a segunda dependerá muito mais do valor pressuposto para a velocidade do primeiro ponto. A velocidade do último ponto é, enfim, completamente determinada pelas velocidades dos $n-1$ outros, já que em todo sistema a quantidade de força viva [energia cinética] se mantém constante. [3. p. 80-81]

Trata-se, portanto, de um gás formado de moléculas monoatômicas ["pontos materiais"] em equilíbrio térmico num campo de forças. Aqui, a hipótese agora utilizada é a de correlação entre as moléculas que colidem, o que fica evidenciado na sua afirmação de que a velocidade da segunda molécula dependerá do valor pressuposto para a velocidade da primeira.

Em seguida ele nos apresenta mais uma vez (ele já o tinha feito no início da primeira seção do artigo) sua definição da noção de probabilidade enquanto média temporal, identificando-a com o tempo relativo que uma molécula tem velocidade num dado intervalo no espaço das velocidades.

Eu quero, portanto, designar o tempo durante o qual as velocidades estão distribuídas de tal maneira que no decorrer do tempo, $c_{2}$ está entre $c_{2}$ e $c_{2}+d c_{2}$, $c_{3}$ entre $c_{3}$ e $c_{3}$ e $c_{3}$ e $c_{3}+d c_{3}$ e assim por diante até $c_{n}$, ou seja, a probabilidade da distribuição das velocidades, como $\varphi\left(c_{2}, c_{3} \ldots, c_{n}\right) d c_{2} d c_{3} \ldots d c_{n}$. A probabilidade de que $c_{1}$ esteja entre $c_{1}$ e $c_{1}+d c_{1}$ e as velocidades restantes, exceto $c_{2}$, estejam entre os limites análogos, seria:

$$
\varphi\left(c_{1}, c_{3}, c_{4}, \ldots, c_{n}\right) d c_{1} d c_{3} \ldots d c_{n}
$$

e assim por diante. [3, p. 81]

Ainda no mesmo contexto de significados, ele assume a existência de uma quantidade total fixa de energia que será distribuída entre as diversas moléculas, de tal forma que as combinações de energia são igualmente prováveis.

Além disso, as forças vivas [energias cinéticas] seriam

$$
\frac{m_{1} c_{1}^{2}}{2}=k_{1}, \quad \frac{m_{2} c_{2}^{2}}{2}=k_{2} \quad \ldots \frac{m_{n} c_{n}^{2}}{k_{n}}=k_{n},
$$

e a probabilidade de que $k_{2}$ esteja entre $k_{2} \mathrm{e}$ $k_{2}+d k_{2}, k_{3}$ entre $k_{3}$ e $k_{3}+d k_{3} \ldots k_{n}$ entre

$$
\begin{aligned}
& k_{n} \text { e } k_{n}+d k_{n} \text { seria } \\
& \qquad \psi_{1}\left(k_{2}, k_{3} \ldots k_{n}\right) d k_{2} d k_{3} \ldots d k_{n}
\end{aligned}
$$

Seria análoga a interpretação de $\psi_{2}\left(k_{1}, k_{2} \ldots k_{n}\right)$ e assim por diante, de modo que temos

$$
\begin{gathered}
m_{2} c_{2} . m_{3} c_{3} \ldots m_{n} c_{n} \psi_{1}\left(\frac{m_{2} c_{2}^{2}}{2}, \frac{m_{3} c_{3}^{2}}{2} \ldots \frac{m_{n} c_{n}^{2}}{2}\right)= \\
\varphi_{1}\left(c_{2} c_{3} \ldots c_{n}\right)
\end{gathered}
$$

$\mathrm{Ou}$

$$
\begin{aligned}
& \varphi_{1}\left(c_{2}, c_{3} \ldots c_{n}\right)=2^{\frac{n-1}{2}} \sqrt{m_{2} m_{3} \ldots m_{n}} \\
& \sqrt{k_{2} k_{3} \ldots k_{n}} \psi_{1}\left(k_{2}, k_{3} \ldots k_{n}\right)
\end{aligned}
$$

e da mesma forma para os $\varphi$ e $\psi$ restantes.

[3, p. 81]

$\mathrm{Na}$ seqüência do seu raciocínio, ele passa a considerar (a exemplo da primeira seção) uma colisão entre um par de moléculas $m_{r}$ e $m_{s}$, momento em que ele propõe a substituição da condição de não correlação entre as velocidades das moléculas (utilizada na primeira seção) pela correlação entre elas.

Vamos considerar uma colisão entre um par qualquer de pontos [moléculas], por exemplo $m_{r}$ e $m_{s}$, que ocorre de maneira que $c_{r}$ encontra-se entre $c_{r}$ e $c_{r}+d c_{r}$ e $c_{s}$ entre $c_{s}+d c_{s}$. Os limites das mesmas grandezas depois da colisão seriam $c_{r}^{\prime}$ e $c_{r}^{\prime}+d c_{r}^{\prime}$ e $c_{s}^{\prime}$ e $c_{s}^{\prime}+d c_{s}^{\prime} .(\ldots)$ A quantidade dos pontos que se unem entre determinados limites mais precisamente de modo que ao mesmo tempo as velocidades dos pontos restantes se encontrem entre $c_{2}$ e $c_{2}+d c_{2} \ldots$ diferencia-se no entanto daquela do problema anterior pelo simples fato da função $\varphi_{1}\left(c_{2}, c_{3}, \ldots c_{n}\right) d c_{2} d c_{3} \ldots d c_{n}$ ser colocada no lugar do produto $\varphi\left(c_{r}\right) \varphi\left(c_{s}\right) d r d s$. Podese, portanto, no lugar da condição colocada anteriormente

$$
\frac{\varphi\left(c_{r}\right) \cdot \varphi\left(c_{s}\right)}{c_{r} \cdot c_{s}}=\frac{\varphi\left(c_{r}^{\prime}\right) \cdot \varphi\left(c_{s}^{\prime}\right)}{c_{r}^{\prime} \cdot c_{s}^{\prime}}
$$

colocar a condição

$$
\frac{\varphi_{1}\left(c_{2}, c_{3}, \ldots c_{n}\right)}{c_{r} c_{s}}=\frac{\varphi\left(c_{2} \ldots c_{r}^{\prime} \ldots c_{s}^{\prime} \ldots c_{n}\right)}{c_{r}^{\prime} c_{s}^{\prime}} .
$$

Naturalmente o mesmo também vale para $\varphi_{2}, \varphi_{3} \ldots$ todas as vezes que

$$
k_{r}+k_{s}=k_{r}^{\prime}+k_{s}^{\prime}
$$


Portanto, a hipótese de correlação entre as moléculas estava, desde o princípio, no horizonte de trabalho de Boltzmann, que posteriormente vai perceber que ela não lhe permite o enfrentamento do problema da irreversibilidade macroscópica, optando, portanto pela hipótese de não correlação em seu artigo de 1872 .

Mas Boltzmann sente ainda a necessidade de encontrar o tempo durante o qual a energia cinética de uma dada molécula específica encontra-se entre certos limites, $k$ e $k+d k$, sem levar em conta a energia das outras moléculas. É neste momento que ele nos apresenta um método novo, o método combinatorial.

\subsection{O método combinatorial}

O método combinatorial é independente de quaisquer afirmações sobre colisões entre as moléculas e não se baseia em nenhum argumento de natureza cinética. Assumindo que a probabilidade de que uma molécula seja encontrada em uma dada região do espaço é proporcional ao "tamanho" daquela região ele pôde reconstruir os resultados usuais do equilíbrio térmico.

Embora este método não tenha sido bem sucedido na derivação da distribuição de Maxwell em três dimensões, ele é extremamente valioso para a teoria de probabilidades e para a física estatística [14, p. 486].

A idéia central por detrás deste método é que a descrição macroscópica de um estado de equilíbrio (macroestado) não distingue entre os muitos estados microscópicos (microestado) que são compatíveis com ele.

O que Boltzmann fez foi considerar um sistema (um gás composto de um número $n$ muito grande, mas finito, de moléculas) com energia total $E$ dividida em $k$ pedaços discretos $x$, de tal modo que $E=\sum_{i} k_{i}=n x$, onde $k_{i}$ é a energia da $i$-ésima molécula ${ }^{12}$.

Em termos gerais, o problema com o qual Boltzmann se deparou foi o seguinte:

Qual é a probabilidade de que a energia de uma molécula esteja entre $k$ e $k+d k$ independente das energias das outras moléculas do sistema?

Sua intenção era derivar uma expressão para o número dos diferentes caminhos possíveis de dividir a quantidade total de energia entre as diferentes moléculas.

Seu ponto de partida na busca de solução do problema foi dividir a energia total $n x$ do sistema em $p$ partes iguais, de modo que o contínuo dos valores de energia de cada uma das moléculas fosse dividido em um número finito de intervalos.

Para isso vamos dividir toda a força viva $n x$ contida no sistema em infinitas partes iguais p. [3, p. 84]
Este procedimento está em acordo com o vínculo da energia que ele estabelece anteriormente, ou seja, $E=\sum_{i} k_{i}=n x$.

Na sequência do seu raciocínio, ele exemplifica esse procedimento tratando o caso em que existem apenas duas moléculas no sistema $(n=2)$, ocasião em que existirão $p$ possíveis maneiras de dividir a energia entre elas.

E se no caso de existirem apenas dois pontos materiais, ou seja, para $n=2$, a probabilidade de $k_{1}$ estar dentro de qualquer um dos p intervalos de 0 até $\frac{2 x}{p}, \frac{2 x}{p}$ até $\frac{4 x}{p}, \frac{4 x}{p}$ até $\frac{6 x}{p}$ e assim por diante, for igual, o problema estará resolvido. 3, p. 84]

Neste caso, devido à restrição estabelecida pela energia total, se a primeira molécula está no $p$-ésimo intervalo, a segunda molécula deve estar no primeiro intervalo; se a primeira molécula está no $(p-1)$-ésimo intervalo, a segunda molécula deve estar no segundo intervalo; e assim por diante. Nesse contexto, Boltzmann supõe que todos esse possíveis casos têm a mesma probabilidade (postulado de "igualdade de probabilidades a priori"). Portanto, Boltzmann constrói um "espaço amostral dos estados" e utiliza como "argumento heurístico" a "equiprobabilidade dos estados".

Em seguida ele discute o caso em que o sistema é composto de três moléculas, ou seja, $(n=3)$.

Para $n=3$, é necessário que, se $k_{1}$ estiver entre $(p-1) \frac{3 x}{p}$ e $p \cdot \frac{3 x}{p}$, tanto $k_{2}$ quanto $k_{3}$ estejam dentro do primeiro dos $p$ intervalos. Se $k_{1}$ estiver dentro do penúltimo intervalo, ou seja, para

$$
(p-2) \frac{3 x}{p} \leq k_{1} \leq(p-1) \frac{3 x}{p},
$$

há dois casos possíveis; pode ser que $k_{2}$ caia no primeiro ou no segundo dos $p$ intervalos e $k_{3}$ será sempre definido. Para

$$
(p-3) \frac{3 x}{p} \leq k_{1} \leq(p-2) \frac{3 x}{p},
$$

haverá três intervalos possíveis para $k_{2}$ e assim por diante. Ora, já que todos esses casos são provavelmente iguais e seu número total é

$$
1+2+3 \ldots=\frac{p(p+1)}{2},
$$

então a probabilidade de $k_{1}$ estar entre $(p-q) \frac{3 x}{p}$ e $(p-q) \frac{3 x}{p}+\frac{3 x}{p}$ será $\frac{2 q}{p(p+1)}$, onde uma vez que $p$ é infinito, pode-se colocar $\frac{2 q}{p^{2}}$. Vamos estabelecer $(p-q) \frac{3 x}{p}=k_{1}$ e $\frac{3 x}{p}=d k_{1}$, e a probabilidade de $k_{1}$ estar entre $k_{1}$ e $k_{1}+d k_{1}$ será $\frac{2\left(3 x-k_{1}\right) d k_{1}}{3 x^{2}}$. [3, p. 84]

\footnotetext{
${ }^{12}$ Esta postura eminentemente finitista de Boltzmann sempre esteve em concordância com a sua intuição física. Essa visão será retomada em seu artigo de 1872, quando da construção de um interessante método de trabalho baseado na discretização da energia.
} 
Neste caso, se uma molécula está em um dado estado $s$, onde $s=1,2, \ldots, p$, as outras duas moléculas têm energia total igual a $q=p-s$. Portanto, existem $q$ possibilidades para a energia cinética da primeira molécula estar no intervalo (estado) $s=p-q$. É neste momento que Boltzmann nos apresenta uma segunda concepção da noção de probabilidade, concebida agora como a razão entre o número de casos favoráveis e o número de casos possíveis, ou seja, a probabilidade de que uma dada molécula tenha energia " $k_{i} x$ " é definida como o número de microestados para os quais a partícula $i$ tem esta quantidade de energia dividida pelo número total de microestados ${ }^{13}$. Esta é a chamada média no ensemble de partículas. Consequentemente, a probabilidade de que uma molécula esteja no $s$-ésimo intervalo é $\frac{2 q}{p(p+1)}$. Como $p$ é muito grande, esta probabilidade pode ser escrita como $\frac{2 q}{p^{2}}$.

Fazendo $(p-q) \frac{3 x}{p}=k$ e $\frac{3 x}{p}=d k$, a expressão para a probabilidade de que uma molécula seja encontrada com energia no intervalo entre $k$ e $k+d k$ torna-se

$$
P_{3}(k) d k=\frac{2(3 x-k) d k}{(3 x)^{2}},
$$

como escreve Boltzmann.

Para o caso em que $n=4$ (sistema composto de quatro moléculas), ele diz:

Vamos supor $n=4$ e, estando $k_{1}$ no último intervalo, ou seja, $(p-1) \frac{4 x}{p} \leq k_{1}$, só um caso será possível, que $k_{2}$ e $k_{3}$ estejam no primeiro intervalo. Sendo

$$
(p-2) \frac{4 x}{p} \leq k_{1} \leq(p-1) \frac{4 x}{p},
$$

três casos serão possíveis, os intervalos 11 , 12 e 21 para $k_{2}$ e $k_{3}$; para:

$$
(p-3) \frac{4 x}{p} \leq k_{1} \leq(p-2) \frac{4 x}{p},
$$

já há seis casos possíveis para $k_{2}$ e $k_{3}$, os intervalos 11, 12, 13, 21, 22 e $31 ; k_{4}$ é sempre definido. Em geral, para

$$
(p-q) \frac{4 x}{p} \leq k_{1} \leq(p-q+1) \frac{4 x}{p},
$$

serão possíveis $(p-q) \frac{4 x}{p}$ casos. É fácil se convencer disso tomando-se, no lugar dos intervalos para $k_{2}$ e $k_{3}$, os intervalos para $k_{2}$ e $k_{2}+k_{3}$; os mesmos seriam, no primeiro caso, 11 , no segundo, $11,12,22$, mas no terceiro $11,12,13,22,23,33$, ou seja nada mais que combinação de ambos, com repetição dos elementos 1, 2, 3... Para todos os valores de $k_{1}$ resultam ao todo

$$
\frac{1.2}{2}+\frac{2.3}{2} \ldots \frac{p(p+1)}{2}=\frac{p(p+1)(p+2)}{2.3},
$$

casos. Portanto, a probabilidade de $k_{1}$ estar entre $(p-q) \frac{4 x}{p}$ e $(p-q) \frac{4 x}{p}+\frac{4 x}{p}$ será $\frac{3 q(q+1)}{p(p+1)(p+2)}$, não considerando o infinitamente pequeno, temos $\frac{3 q^{2}}{p^{3}}$. Se colocarmos novamente $(p-q) \frac{4 x}{p}=k_{1}$ e $\frac{4 x}{p}=d k_{1}$, para a probabilidade de $k_{1}$ estar entre $k_{1}$ e $k_{1}+d k_{1}$ obteremos: $\frac{3\left(4 x-k_{1}\right)^{2} d k_{1}}{(4 x)^{3}}$. [3, p. 84-85]

Aqui, Boltzmann nos apresenta o número de casos possíveis como sendo

$$
\frac{p(p-1)(p+2)}{3 !},
$$

e o número de casos favoráveis como

$$
\frac{q(q+1)}{2}
$$

o que nos dá a seguinte expressão para a probabilidade de encontramos $k$ entre $k k+d k$

$$
\frac{3 q(q+1)}{p(p+1)(p+2)}
$$

que, a partir das mesmas substituições anteriores para $k$ e $d k$, nos dá

$$
P_{4}(k) d k=\frac{3(4 x-k)^{2} d k}{(4 x)^{3}} .
$$

No caso mais geral, aquele em que se considera as $n$ moléculas, com energia total $n x$, a probabilidade de $k$ estar entre $k$ e $k+d k$ é dada por

$$
P_{n}(k) d k=\frac{(n-1)(n x-k)^{n-2} d k}{(n x)^{n-1}} .
$$

Tomando o limite quando $n \rightarrow \infty$ (limite termodinâmico), Boltzmann chega finalmente a

$$
\frac{1}{x} e^{-\frac{k}{x} d k}
$$

Colocando $\frac{m c^{2}}{2}$ no lugar de $k$, ele obteve exatamente a expressão para a distribuição da energia de uma molécula em duas dimensões.

Com o argumento de "igualdade de probabilidades a priori", Boltzmann deixa claro por que um sistema em equilíbrio deveria obedecer a lei de distribuição de Maxwell. Simplesmente porque ela é a mais provável

\footnotetext{
${ }^{13}$ Os microestados são definidos pela designação de $k_{1}$ pedaços de energia para a partícula 1 , $k_{2}$ pedaços de energia para a partícula $2 \ldots k_{n}$ pedaços de energia para a partícula $n$, onde $\sum_{i} k_{i}=k$.
} 
de ser encontrada no equilíbrio térmico, uma vez que ela corresponde ao maior número de microestados.

A hipótese de igualdade de probabilidades, uma distribuição uniforme de probabilidades no espaço dos estados, nos conduziu posteriormente, através dos trabalhos de Gibbs à definição de "ensemble microcanônico", um conjunto de estados microscópicos, caracterizados por um mesmo valor constante da energia, aos quais se associam iguais pesos probabilísticos.

\section{A dupla interpretação da função de distribuição de Maxwell e a noção de probabilidade de estado}

Como observado por M.J. Klein [16, p. 62], e a discussão do método cinético e combinatorial no item anterior pôde comprová-lo, em sua análise da natureza do equilíbrio termodinâmico, Boltzmann interpreta a função de distribuição de Maxwell em dois diferentes caminhos, que ele parece considerar como equivalentes e que estão diretamente associados a noção mesma de probabilidade, concebida como:

1. Média no tempo

Identificada como a fração de um intervalo de tempo suficientemente longo, durante o qual a velocidade de uma molécula específica tem valores dentro de um certo elemento de volume no espaço das velocidades.

2. Média no ensemble de partículas

Identificada como a fração do número total de moléculas que, num dado instante, tem velocidades num dado elemento de volume.

Mas Boltzmann também usou o conceito de probabilidade para se referir ao estado de um gás como um todo, momento em que ele introduz o conceito de probabilidade de estado de um gás.

$\mathrm{Na}$ terceira seção do seu artigo de 1868, onde ele nos apresenta uma solução geral para o problema do equilíbrio térmico, seu ponto de partida foi considerar um sistema de $n$ pontos materiais, representando suas coordenadas e componentes das velocidades respectivamente por $\left(x_{i}, y_{i}, z_{i}\right)$ e $\left(u_{i}, v_{i}, w_{i}\right)$, onde $\left.i=1,2, \ldots, n\right)$.

Se nós caracterizamos a posição do ponto $m_{1}$ através de três coordenadas retangulares $\left(x_{1}, y_{1}, z_{1}\right)$, do ponto $m_{2}$ através de $\left(x_{2}, y_{2}, z_{2}\right)$ etc... e as três componentes das velocidades $c_{1}$ do ponto $m_{1}$ pelas coordenadas $\left(u_{1}, v_{1}, w_{1}\right)$, as correspondentes componentes do segundo ponto $\left[m_{2}\right]$ de $\left(u_{2}, v_{2}, w_{2}\right)$ etc. [3, p. 92]

Em seguida, utilizando sua primeira definição de probabilidade como média temporal [inicialmente utilizada no contexto de uma molécula], ele introduz a probabilidade de um certo estado do sistema como a proporção relativa de tempo no qual o gás permanece numa dada região do espaço, o que é feito através da indicação da probabilidade de que os parâmetros do gás assumam valores em certos intervalos.

e marcamos o tempo, simultaneamente, no curso de um tempo muito longo, segundo o qual $x_{1}$ está entre $x_{1}$ e $x_{1}+d x_{1}, y_{1}$ entre $y_{1}$ e $y_{1}+d y_{1}, z_{1}$ entre $z_{1}$ e $z_{1}+d z_{1}$, portanto, $m_{1}$ está dentro do elemento de volume $d s_{1}=d x_{1} d y_{1} d z_{1}$, além disso $m_{2}$ da mesma forma, dentro do elemento de volume $d s_{2}=d x_{2} d y_{2} d z_{2} \ldots m_{n}$ dentro do elemento de volume $d s_{n}=d x_{n} d y_{n} d z_{n}$, e ainda $u_{1}$ dentro dos limites $u_{1}$ e $u_{1}+d u_{1}$, $v_{1}$ dentro de $v_{1}$ e $v_{1}+d v_{1}, w_{1}$ dentro de $w_{1}$ e $w_{1}+d w_{1}$; portanto, no final [no equilíbrio] $c_{1}$ estará dentro do elemento de volume $d \sigma_{1}=d u_{1} d v_{1} d w_{1}, c_{2}$ dentro do elemento de volume $d \sigma_{2}=d u_{2} d v_{2} d w_{2} \ldots$, $c_{n-1}$ dentro do elemento de volume $d \sigma_{n-1}=$ $d u_{n-1} d v_{n-1} d w_{n-1}$, e finalmente o ponto $c_{n}$, que estará dentro do elemento de volume $d \sigma_{n}=d u_{n} d v_{n} d w_{n}$, dividido por um tempo muito longo de forma que

$$
\begin{aligned}
& f\left(x_{1}, y_{1}, z_{1} \ldots x_{n}, y_{n}, z_{n}, u_{1}, v_{1}, w_{1} \ldots\right. \\
& \left.u_{n-1} v_{n-1} w_{n-1}, u_{n} v_{n} w_{n}\right) d x_{1} d y_{1} d z_{1} \ldots \\
& d z_{n} d u_{1} \ldots d u_{n-1} d v_{n-1} d w_{n-1} d w-n .
\end{aligned}
$$$$
\text { [3, p. 92-93] }
$$

Portanto, a probabilidade de um certo estado do gás em um instante de tempo $t$ é representada por

$$
f\left(x_{i}, y_{i}, z_{i} \ldots ; u_{i}, v_{i}, w_{i}\right) d x_{i} d y_{i} d z_{i} d u_{i} d v_{i} d w_{i},
$$

onde $f$ é uma função dos parâmetros do movimento e $d w$ é o produto da diferencial desses parâmetros.

Assim, diferente de 1866, Boltzmann não fala mais em trajetória de uma partícula, mas dos limites das coordenadas de posição e velocidade do conjunto delas.

As probabilidades, ensaiadas conceituamente na abordagem cinética de 1866 e utilizadas implicitamente em sua dupla significação em 1868, vão aparecer de maneira explícita em 1871 através da expressão "a probabilidade dos diferentes estados dos corpos" ("die Wahrscheinlichkeit der verschiedenen Zustände des Körpers"), e estarão subentendidas na expressão "distribuição de estado" ("Zustandsverteilung").

Em 1871, em Sobre o Equilíbrio num Gás Composto de Moléculas Poliatômicas [5], Boltzmann vai generalizar a função de distribuição de velocidades moleculares de Maxwell, considerando agora o caso de um gás em equilíbrio termicc ${ }^{14}$, composto de moléculas poliatômicas sujeitas a um potencial. Seu ponto de partida foi, naturalmente, considerações em torno da expressão da função de distribuição derivada por Maxwell

\footnotetext{
${ }^{14}$ Posteriormente, em seu artigo de 1872 Boltzmann colocará em questão o equilíbrio térmico como ponto de partida e desenvolverá um estudo da rota para o equilíbrio, momento em que surgirá a sua famosa equação de transporte, conhecida como "equação de Boltzmann".
} 
em 1867. Como ponto de partida de sua análise, Boltzmann reconhece que os gases encontrados na natureza (gases reais) têm uma estrutura mais complexa do que aquela até então considerada ${ }^{15}$.

No entanto, os gases que se encontram na natureza certamente não são pontos materiais isolados. Nós chegaremos mais perto da verdade se nós os compreendermos como um sistema de diversos pontos materiais (os assim chamados átomos) que, através de certas forças, são mantidos juntos. O estado de uma molécula em um dado instante de tempo, de modo algum depende de uma única variável, mas de diversas variáveis. 5 , p. 238

Ele então nos apresenta a sua estratégia para a resolução do problema de caracterização do estado de uma dada molécula num certo instante de tempo.

Para pensar o estado de uma molécula num dado instante de tempo $t$, nós imaginamos três direções perpendiculares uma a outra em cada região fixa considerada. Nós traçamos três eixos coordenados perpendiculares paralelos aquelas três direções no ponto no qual se encontra o centro de gravidade de nossa molécula no tempo $t$, e fazemos as coordenadas dos seus pontos materiais com relação aqueles eixos no momento $t$ serem

$$
\xi_{1}, \eta_{1}, \varsigma_{1}, \xi_{2}, \eta_{2}, \zeta_{2} \ldots \xi_{r-1}, \eta_{r-1}, \zeta_{r-1}
$$

O número de pontos materiais de nossa molécula, que nós sempre queremos chamar seus átomos, é $r$. As coordenadas do $r$ ésimo átomo são determinadas por meio das $r-1$ outras, uma vez que o centro de gravidade é o ponto coordenado inicial. Além do mais a velocidade do primeiro átomo é $c_{1} \mathrm{e}$ $u_{1}, v_{1}, w_{1}$ suas componentes na direção dos eixos coordenados; o mesmo deve ser válido para o segundo átomo, $\operatorname{com} c_{2}, u_{2}, v_{2}, w_{2}$; para o terceiro átomo, $c_{3}, u_{3}, v_{3}, w_{3}$ e assim por diante. Portanto, o estado de nossa molécula, num dado instante t, é definido se nós conhecemos os valores das $6 r-3$ quantidades neste tempo. [5, p. 238, grifos nossos]

A noção de estado de uma molécula recebe de Boltzmann uma nova representação, que na sequência do seu raciocínio, ao considerar um conjunto de moléculas, será estendida para o gás como um todo, a partir da caracterização do estado de uma fração $(d N)$ do total de moléculas $(N)$.
As coordenadas do centro de gravidade de nossa molécula com respeito aos eixos coordenados não determinam assim os estados, mas tão somente a sua localização. Nós agora queremos dizer abreviadamente que uma molécula está em uma certa região se seu centro de gravidade está naquela região e assumir que, em média, $N$ moléculas estão no volume considerado. Destas $N$ moléculas somente uma pequena parte $d N$, num dado instante de tempo $t$, terá simultaneamente coordenadas do primeiro átomo entre:

$\xi_{1}$ e $\xi_{1}+d \xi_{1}, \eta_{1}$ e $\eta_{1}+d \eta_{1}, \zeta_{1}$ e $\zeta_{1}+d \zeta_{1}$,

do segundo átomo entre

$\xi_{2}$ e $\xi_{2}+d \xi_{2}, \eta_{2}$ e $\eta_{2}+d \eta_{2}, \zeta_{2}$ e $\zeta_{2}+d \zeta_{2}$,

do $r$-1-ésimo átomo entre

$\xi_{r-1}$ e $\xi_{r-1}+d \xi_{r-1}, \eta_{r-1}$ e $\eta_{r-1}+d \eta_{r-1}$, $\zeta_{r-1}$ e $\zeta_{r-1}+d \zeta_{r-1}$,

além disso as componentes da velocidade do primeiro átomo entre

$u_{1}$ e $u_{1}+d u_{1}, v_{1}$ e $v_{1}+d v_{1}, w_{1}$ e $w_{1}$ e $w_{1}+d w_{1}$,

do segundo átomo entre

$u_{2}$ e $u_{2}+d u_{2}, v_{2}$ e $v_{2}+d v_{2}, w_{2}$ e $w_{2}$ e $w_{2}+d w_{2}$,

do $r$-ésimo átomo entre

$u_{r}$ e $u_{r}+d u_{r}, v_{r}$ e $v_{r}+d v_{r}, w_{r}$ e $w_{r}$ e $w_{r}+d w_{r}$.

Eu quero chamar de $(A)$ o estado destas moléculas que estão nos limites descritos brevemente acima. Inicialmente ele é

$$
\begin{aligned}
d N= & f\left(\xi_{1}, \eta_{1} \ldots \zeta_{r-1}, u_{1}, v_{1} \ldots w_{r}\right) \\
& d \xi_{1} d \eta_{1} \ldots d \zeta_{r-1} d u_{1} d v_{1} \ldots d w_{r} . \\
& \text { [5, p. 238-239] }
\end{aligned}
$$

Portanto, $d N$ representa o estado das moléculas num dado instante $t$, caracterizado pelo número delas que, no instante considerado, se encontram em determinados limites. Neste sentido, a função $f\left(\xi_{1}, \eta_{1} \ldots \zeta_{r-1}, u_{1}, v_{1} \ldots w_{r}\right)$ é utilizada como ferramenta heurística na representação da probabilidade de um certo estado do gás em um dado instante de tempo.

\section{A probabilidade dos diferentes estados das moléculas seria conhecida se nós soubéssemos que valores para cada gás esta função assume se o mesmo estivesse com temperatura e densidade constantes. [5. p. 239]}

Boltzmann compara, então, seu trabalho com o estudo de Maxwell, desenvolvido para um gás monoatômico.

\footnotetext{
${ }^{15}$ Aqui se incluem as análise de Maxwell em 1867 e do próprio Boltzmannn em 1868.
} 
Para gases cujas moléculas têm um único átomo, Maxwell encontrou que a função $f$ assume o valor:

$$
4 \sqrt{\frac{h^{2}}{\pi}} N e^{-h c^{2}} c^{2} d c
$$

A determinação desta função para gases cujas moléculas são compostas por mais de um átomo parece muito difícil, uma vez que nós não somos capazes de integrar as equações de movimento para um complexo de três átomos. Contudo nós veremos que, a partir das equações de movimento, sem sua integração, nós podemos encontrar um valor para a função $f$, que representa as moléculas através do movimento destas. 5, p. 239240]

$\mathrm{Na}$ seqüência do seu raciocínio, Boltzmann reconhece que, com o passar do tempo, em função do processo de colisão entre as moléculas, a função $f$ assumirá diferentes valores, de modo que ao final de um certo intervalo de tempo $t+\delta t$ ela deverá ser representada diferentemente, por exemplo por $f_{1}\left(\xi_{1}^{\prime}, \eta_{1}^{\prime} \ldots \zeta_{r-1}^{\prime}, u_{1}^{\prime}, v_{1}^{\prime} \ldots w_{r}^{\prime}\right)$. Mas as atenções de Boltzmann estavam voltadas para o equilíbrio térmico, portanto, ele estava principalmente interessado no comportamento do gás que deixa a função $f$ invariável no tempo. Assim ele estabelece como representação da condição de equilíbrio a situação em que

$$
\begin{aligned}
& f_{1}\left(\xi_{1}^{\prime}, \eta_{1}^{\prime} \ldots \zeta_{r-1}^{\prime}, u_{1}^{\prime}, v_{1}^{\prime} \ldots w_{r}^{\prime}\right)= \\
& f\left(\xi_{1}, \eta_{1} \ldots \zeta_{r-1}, u_{1}, v_{1} \ldots w_{r}\right) .
\end{aligned}
$$

Na seqüência do seu raciocínio, ele se detém na análise da colisão entre moléculas de gases de dois tipos diferentes, que ele caracteriza de $G$ e $G^{*}$. Ele assume que num dado instante de tempo $t$ o número de moléculas do gás $G$, que se encontram num dado elemento de volume, anteriormente caracterizado, é

$$
d N=f\left(\varphi_{1}, \varphi_{2}, \ldots\right) d \xi_{1} d \eta_{1} \ldots d w_{r}
$$

onde $\varphi_{1}$ é função de $\left(\xi_{1}, \eta_{1} \ldots w_{r}\right), \varphi_{2}$ é função de $\left(\xi_{1}, \eta_{1} \ldots w_{r}\right)$ e assim por diante.

Da mesma forma o número de moléculas do gás $G^{*}$ pode ser escrito como

$$
d N^{*}=f^{*}\left(\varphi_{1}^{*}, \varphi_{2}^{*}, \ldots\right) d \xi_{1}^{*} d \eta_{1}^{*}, \ldots d w_{r}^{*}
$$

Em cada um dos casos a função de distribuição é escrita respectivamente como

$$
\begin{aligned}
& f=A e^{-h \varphi} \\
& f^{*}=A^{*} e^{-h \varphi^{*}}
\end{aligned}
$$

onde $A, A^{*}$ e $h$ são constantes e $\varphi$ representa a soma das energias cinéticas das moléculas.

Assim, o número de moléculas nos limites $A$ do gás $G$ pode ser reescrita como

$$
d N=A e^{-h \varphi} d \xi_{1} d \eta_{1} \ldots d_{r-1} d u_{1} d v_{1} \ldots d w_{r},
$$

onde:

$$
A=\frac{N}{\iint \ldots e^{-h \varphi} d \xi_{1} d \eta_{1} \ldots d w_{r}} .
$$

Boltzmann chega então ao ponto crucial da sua generalização, momento em que ele incorpora a função potencial.

Nós agora queremos olhar para a conseqüência mais importante da distribuição encontrada. Se nós representamos a função força entre os átomos das moléculas, desde que a mesma não se choque com uma outra, por $\chi$ e as massas dos átomos por $m_{1}, m_{2}, \ldots m_{r}$ suas respectivas velocidades por $c_{1}, c_{2}, \ldots, c_{r}$, então

$$
\varphi=\chi+\frac{m_{1} c_{1}^{2}}{2}+\frac{m_{2} c_{2}^{2}}{2}+\ldots \frac{m_{r} c_{r}^{2}}{2},
$$

portanto, a fórmula 21 [aqui indicada na Eq. (22)] fica

$$
\begin{aligned}
& d N=A e^{-h\left(\chi+\frac{m_{1} c_{1}^{2}}{2}+\frac{m_{2} c_{2}^{2}}{2}+\ldots\right)} \\
& d \xi_{1} d \eta_{1} \ldots d w_{r} . \\
& {[5 \text {, p. 256] }}
\end{aligned}
$$

Com esta expressão Boltzmann nos mostra que a probabilidade dos diferentes estados de uma molécula não depende unicamente da natureza das outras moléculas, com as quais aquela primeira interage, mas também da temperatura e da constante $h$.

Em seguida ele conclui indicando-nos o caminho para se calcular o valor médio de qualquer função das coordenadas e velocidades das moléculas.

Por meio da fórmula 23 [expressão para $d N$ na última citação apresentada], nós imediatamente podemos encontrar o valor médio de cada uma das funções das coordenadas e velocidades dos átomos de nossas moléculas através do simples ajuste; seja $X$ uma tal função, $\bar{X}$ seu valor médio, assim,

$$
\bar{X}=\frac{1}{N} \int X d N
$$

onde a integração é feita sobre todos os valores possíveis das variáveis contidas em $d N$. [5. p. 256]

Como exemplo de utilização do cálculo do valor médio, Boltzmann calcula a energia cinética média de um átomo. 
Para a energia cinética média de um átomo, por exemplo o primeiro, nós encontramos através do cálculo da integração

$$
\frac{m_{1}}{2} \overline{c_{1}^{2}}=\frac{1}{N} \int \frac{m_{1} c_{1}^{2}}{2} d N=\frac{3}{2 h} .[5, \text { p. } 257]
$$

Em uma notação moderna $\frac{3}{2 h}$ é igual a $\frac{3}{2} K T$, onde $T$ é a temperatura absoluta e $K$ é a constante de Boltzmann.

Logo em seguida Boltzmann observa que a energia cinética média é a mesma para todos os átomos, sendo considerada como medida da temperatura dos mesmos.

A energia cinética média é a mesma para todos os átomos das moléculas em mútua interação. Nós chamamos o estado térmico, que muitos átomos assumem na interação, o estado de mesma temperatura; com a mesma temperatura a energia cinética média de cada átomo é a mesma; ela pode ser considerada a medida da temperatura.

[5, p. 257]

Neste sentido, o equilíbrio termodinâmico fica caracterizado a partir da igualdade de temperatura.

A probabilidade, concebida como a fração de tempo durante a qual o sistema permanece num dado estado, aparece de maneira bastante explícita logo no início de um outro artigo também publicado em 1871. Tratase de Demonstração Analítica da $2^{a}$ lei da Teoria Mecânica do Calor a partir do Teorema sobre o Equilíbrio da Força Viva [4]. Nele Boltzmann afirma:

Deixemos as condições exteriores (de temperatura e da força externa atuante), sob as quais o corpo se encontra permanecerem imutáveis durante um tempo $T$ bastante longo, de forma que nós designaremos por $\tau$, cada fração de $T$ durante a qual $x_{1}, y_{1} \ldots z_{r}$; $u_{1}, v_{1} \ldots w_{r}$ estão simultaneamente contidos na fronteira

$$
\begin{aligned}
& \text { (A) } \quad x_{1} \quad \text { e } \quad x_{1}+d x_{1}, y_{1} \quad \text { e } \\
& y_{1}+d y_{1} \ldots w_{r} \quad \text { e } \quad w_{r}+d w_{r} \text {. }
\end{aligned}
$$

A proporção $\frac{\tau}{T}$ é por mim definida como o tempo segundo o qual, em média, o estado dos corpos permanecem encerrados nas fronteiras de (A). [4, p.288]

É explícita nesta passagem de Boltzmann a caracterização do estado dos corpos em função dos limites das coordenadas dos átomos, que são por sua vez caracterizados em termos de probabilidades.

Na seqüência da citação acima, ele formaliza sua definição de probabilidade como a fração de tempo $\frac{\tau}{T}$, durante a qual $\boldsymbol{r}$ átomos em uma molécula tem coordenadas de posição nos intervalos $x_{1}$ e $x_{1}+d x_{1}, y_{1}$ e $y_{1}+d y_{1} \ldots z_{r}$ e $z_{r}+d z_{r}$ com as componentes de velocidade entre $u_{1}$ e $u_{1}+d u_{1}, v_{1}$ e $v_{1}+d v_{1} \ldots, w_{r}$ e $w_{r}+d w_{r}$, ele diz:

Eu tenho encontrado em um tratado anterior, utilizando uma hipótese bem segura, que esta proporção [a fração de tempo $\frac{\tau}{T}$ ], no caso onde os corpos estão em contato com um número infinito de moléculas de um gás, tem o seguinte valor

$$
d t=\frac{e^{-h \varphi} d x_{1} d y_{1} \ldots d z_{1} d u_{1} \ldots d w_{r}}{\int \ldots \int e^{-h \phi} d x_{1} d y_{1} \ldots d z_{1} d u_{1} \ldots d w_{r}}
$$

onde nós temos

$$
\varphi=\chi+\Sigma \frac{m c^{2}}{2} . \quad \text { [4, p. 288-289] }
$$

Vê-se, portanto, que a função de probabilidades presente na distribuição de velocidades de Maxwell, utilizada aqui em sua versão mais geral, é agora utilizada como recurso heurístico, não unicamente para caracterizar a fração do número total de moléculas que, num dado instante, têm velocidades num dado elemento de volume (probabilidade concebida como média no ensemble de partículas), mas também para determinar a fração de tempo segundo a qual o sistema permanece, em média, em um dado estado.

Em Demonstração Analítica da $2^{a}$ lei da Teoria Mecânica do Calor a partir dos Teoremas sobre o Equilíbrio da Força Viva [4, ao definir esta fração de tempo $\left(\frac{\tau}{T}\right)$, cuja derivação formal ele remete a um artigo anterior (ele se refere a Algumas Considerações Gerais Sobre o Equilíbrio Térmico [6]), Boltzmann pôde relacionar formalmente a evolução do sistema no tempo com os limites do movimento das partículas no espaço.

A estratégia foi associar as mudanças de estado às colisões entre as moléculas. Seu ponto de partida foi caracterizar o estado, a partir da consideração do número de átomos cujas coordenadas de posição e velocidade estão compreendidas em determinados limites. Em seguida ele escreve o número de partículas que entram em um dado estado em função das coordenadas de estado de onde elas saem. A idéia aqui é trabalhar sobre os volumes no espaço, pois cada estado é representado pelos limites de suas respectivas coordenadas. Para caracterizar a evolução dos estados no tempo ele busca estabelecer uma equivalência entre os volumes infinitesimais do espaço. Neste momento, a hipótese fundamental de Boltzmann é a de que o número de partículas que entram no estado é igual ao número daquelas que saem do mesmo estado, ou seja, a freqüência das entradas e das saídas num mesmo estado são iguais. Com isso ele pretende justificar a equiprobabilidade dos estados, isto é, que a função que dá a fração de tempo segundo a qual as partículas permanecem em um estado é a 
mesma para todos os estados, dependendo unicamente da energia $(\varphi)$, como nos indica sua expressão para $d t$.

Nós assumiremos que as colisões são tão frequentes que, no momento em que termina uma colisão, uma outra se produz novamente.(...) Segundo esta suposição o número de colisões para as quais as variáveis estão nas fronteiras (E) é igual ao número de colisões para as quais as variáveis estão nas fronteiras iniciais. O primeiro número é a frequência com que as variáveis entram nestas fronteiras, e o último é a freqüência com que elas saem, visto que depois nós supomos, o número de entradas e de saídas por causa do movimento dos átomos dos corpos sem colisão entre as moléculas é negligenciado. [4, p. 289-290]

Em seguida, Boltzmann mostra que a função $e^{-h \varphi}$ satisfaz a condição de igualdade de frequências de entrada e saída dos estados, a partir da qual ele impõem a equiprobabilidade dos diversos estados.

Portanto, as probabilidades aparecem agora como uma nova representação da evolução do sistema, dadas em função das coordenadas de posição e velocidades de cada partícula. É como suporte dessa nova representação que o "espaço de fase" encontra seu lugar, como o espaço de todos os estados acessíveis ao sistema estudado.

\section{Uma abordagem à $2^{\mathrm{a}}$ lei baseada na noção de probabilidades de estado}

Agora, de posse de novos recursos heurísticos, a função de distribuição e a noção de probabilidade de estado, Boltzmann empreende uma nova tentativa de resolução do problema da irreversibilidade presente na $2^{\text {a }}$ lei da termodinâmica. Desta vez ele o faz utilizando uma nova concepção de estado, construída a partir dos resultados obtidos em torno da generalização da função de distribuição de velocidades moleculares de Maxwell. Desta forma ele vai buscar fazer a distinção entre calor e trabalho, o que não tinha sido possível anteriormente, quando o sistema era descrito diretamente por suas variáveis atômicas.

Da mesma forma que em 1866, ele parte da definição de temperatura como a média da energia cinética de um átomo no tempo, que é suposta a mesma para todos os átomos. Em 1871 [5], como visto na seção anterior, ele já havia escrito a expressão para a média desta energia cinética em função de $h$, como

$$
\frac{m}{2} \bar{c}^{2}=\int \frac{m c^{2}}{2} d t=\frac{3}{2 h},
$$

onde $m$ é massa de cada átomo e $c$ sua velocidade.
Em conformidade com uma abordagem estatística, a idéia que se corporifica em Demonstração Analítica da $2^{a}$ lei da Teoria Mecânica do Calor a partir dos Teoremas sobre o Equilíbrio da Força Viva [4] é a de tomar a média sobre toda a distribuição, portanto, sobre o conjunto de $r$ átomos. O valor médio para qualquer função $X(\bar{X})$ do sistema é simplesmente a soma do valor daquela função para cada elemento do espaço de fase, multiplicado pela fração de tempo que o sistema permanece lá, ou seja,

$$
\bar{X}=\int X d t
$$

onde $d t$ é dado por:

$$
d t=\frac{e^{-h \varphi} d x_{1} d y_{1} \ldots d z_{1} d u_{1} \ldots d w_{r}}{\int \ldots \int e^{-h \phi} d x_{1} d y_{1} \ldots d z_{1} d u_{1} \ldots d w_{r}}
$$

sendo $r$ o número de átomos do sistema.

A integral na Eq. (26) fica bastante simplificada para aquelas funções que dependem unicamente das coordenadas de posição ou velocidade, visto que os fatores que envolvem outras coordenadas se cancelam mutuamente, já que aparecem no numerador e no denominador da expressão. Portanto, a função força $(\chi)$, que depende unicamente das coordenadas de posição, é omitida da integral para a energia cinética média de um átomo (Eq. 25). Da mesma forma, as coordenadas de velocidade, estando ausentes da função força, não aparecem na expressão para o valor médio daquela função, que é escrita como

$$
\bar{\chi}=\int \chi d t^{\prime}
$$

onde $d t^{\prime}$ é dado por

$$
d t^{\prime}=\frac{e^{-h \chi} d x_{1} d y_{1} \ldots d z_{r}}{\int \ldots \int e^{-h \chi} d x_{1} d y_{1} \ldots d z_{r}},
$$

Assim é que a temperatura, energia cinética média tomada sobre a distribuição dos $r$ átomos, pôde ser escrita como

$$
T=\overline{\sum \frac{m c^{2}}{2}}=\frac{3 r}{2 h} .
$$

Considerando agora as probabilidades das distribuições ele ataca novamente o problema da irreversibilidade presente na entropia definida por Clausius.

Nós queremos agora proceder à prova de que a diferencial do calor fornecido $\delta Q$, dividida pela energia cinética média,

$$
T=\frac{m}{2} \bar{c}^{2},
$$


de um átomo é sempre uma diferencial exata, e o valor daquela diferencial é determinado analiticamente. Em um artigo apresentado à Academia em 1866, eu tenho já definido a quantidade cuja diferencial é $\frac{\delta Q}{T}$ para o caso de átomos movendo-se juntos em trajetórias fechadas. Se as trajetórias dos átomos não são fechadas, de qualquer modo, é possível encontrar casos especiais para os quais, tanto quanto permitirmos deixar a probabilidade das várias posições atômicas indefinida, $\frac{\delta Q}{T}$ não é uma diferencial exata. Uma extensão exata da prova para este caso é unicamente possível através da consideração daquela probabilidade. 4, p. 295]

Vemos, portanto, que Boltzmann considera a existência de casos especiais nos quais a entropia ("a quantidade cuja diferencial é $\frac{\delta Q}{T}$ ") não é uma diferencial exata, ou seja, uma função dos parâmetros gerais. No entanto, ele reconhece que uma determinação analítica da exatidão daquela diferencial só seria possível introduzindo-se probabilidades, o que é feito quando ele considera a "probabilidade das várias posições" ao invés das posições em si mesmas.

Pode-se assim afirmar que as probabilidades, enquanto recurso heurístico, vão desempenhar aqui um duplo papel:

1. Na descrição do movimento dos átomos

O que lhe permitiu contornar a limitação estabelecida pela periodicidade do movimento dos átomos, associada ao movimento reversível e utilizada quando do uso do seu método cinético de 1866.

2. Na vinculação entre $\frac{\delta Q}{T}$ e o movimento dos átomos

O uso das probabilidades, que como vimos foram construídas a partir da função de distribuição, introduz necessariamente em sua análise, como sublinha Daub [11, p. 324], a função força, o que o levou necessariamente a tratar o problema proposto inicialmente por Clausius, a saber, o do potencial mecânico.

Considerando a energia total $(E)$ fornecida ao corpo como a soma do trabalho necessário para aumentar a energia cinética de cada átomo, mais o trabalho necessário ao deslocamento dos átomos $(\chi)$ ele escreve:

$$
E=\sum \frac{m c^{2}}{2}+\chi
$$

Desta forma a energia média fica sendo:

$$
\bar{E}=\overline{\sum \frac{m c^{2}}{2}}+\bar{\chi}
$$

ou seja,

$$
\bar{E}=\frac{3 r}{2 h}+\bar{\chi}
$$

Como Boltzmann está trabalhando no equilíbrio termodinâmico, alterações infinitesimais na energia média $\bar{E}$, devidas a mudança na distribuição, não afetam a natureza do sistema. As alterações ocorrem através de sucessivos estados de equilíbrio, seja pela alteração da temperatura, que no caso significa alteração da constante $h$, seja pela mudança na energia potencial média do sistema. Assim pode-se escrever

$$
\delta \bar{E}=\delta \frac{3 r}{2 h}+\delta \bar{\chi}
$$

ou seja,

$$
\delta \bar{E}=-\frac{3 r}{2 h^{2}} \delta h+\delta \bar{\chi},
$$

onde $\delta$ representa uma mudança infinitesimal.

Representando o calor fornecido ao sistema em um processo por $\delta^{\prime} Q^{16}$, diferença entre a variação de energia e o trabalho realizado sobre o sistema $\overline{\delta \chi}$, podemos escrever

$$
\delta^{\prime} Q=\delta \bar{E}-\bar{\chi}=-\frac{3 r}{2 h^{2}} \delta h+\delta \bar{\chi}-\overline{\delta \chi}
$$

Portanto, o calor fornecido aos corpos é constituído de três partes, que nas palavras de Boltzmann são:

A primeira, $\delta \overline{\sum \frac{m c^{2}}{2}}$ representa o aumento da força viva [energia cinética] ou elevação da temperatura; a segunda, $\delta \bar{\chi}$, representa o potencial de trabalho interno; e a terceira, $-\overline{\delta \chi}$, que se anula no momento em que a função potencial de todas as forças atuantes sobre os corpos não mudam, representa o calor empregado no potencial de trabalho externo. [4, p. 306-307]

Reescrevendo a Eq. (36), tem-se que

$$
\delta^{\prime} Q=-\frac{3 r}{2 h^{2}} \delta h+\delta\left[\frac{\int \delta \chi e^{-h x} d \sigma}{\int e^{-h x} d \sigma}\right]-\frac{\int e^{-h x} d \sigma}{\int e^{-h x} d \sigma},
$$

onde $d \sigma$ é $d x_{1}, \ldots d z_{r}$.

Como observado na nota de rodapé 15 , a expressão acima não é uma diferencial exata, tendo em vista que $Q$ não é uma função de estado. No entanto Boltzmann mostrou que dividindo esta expressão por $T$ (que é igual a $\left.\frac{3}{2 h}\right)$, nós obtemos uma diferencial exata de uma função, que ele identificou como sendo a entropia de Clausius.

Assim,

$$
\begin{aligned}
\frac{\delta^{\prime} Q}{T}= & -\frac{r \delta h}{h}+\frac{2 h}{3} \delta\left[\frac{\int \chi e^{-h \chi} d \sigma}{\int e^{-h \chi} d \sigma}\right]- \\
& \frac{2 h}{3} \frac{\int \delta \chi e^{-h \chi} d \sigma}{\int e^{-h \chi} d \sigma}
\end{aligned}
$$

cuja integral,

\footnotetext{
${ }^{16} \mathrm{O}$ símbolo $\delta^{\prime}$ é usado aqui para representar uma diferencial inexata, visto que $Q$ não é uma função de estado.
} 


$$
\begin{array}{r}
-r \log h+\frac{2 h}{3} \frac{\int \chi e^{-h \chi} d \sigma}{\int e^{-h \chi} d \sigma}+ \\
\frac{2}{3} \ln \left(\int e^{-h \chi} d \sigma\right)+\text { const. }
\end{array}
$$

pôde finalmente ser identificada com a entropia.

Desta forma Boltzmann obteve o conteúdo essencial da segunda lei para processos reversíveis. Ele calculou ainda, explicitamente, a entropia para um gás monoatômico ideal e para um modelo simples de um corpo sólido. No primeiro caso ele chegou de fato ao resultado conhecido da termodinâmica. No segundo, ele mostrou como a regra de Dulong-Petit poderia ser deduzida.

\section{Conclusão}

Ao longo deste trabalho foi possível investigar alguns instrumentos heurísticos utilizados por Boltzmann em seu programa de pesquisa, desde os seus primeiros estudos em teoria cinética dos gases, motivados pela necessidade de compreensão da irreversibilidade termodinâmica no quadro referencial da mecânica, até uma abordagem dos fenômenos térmicos que caracteriza a emergência de um programa mecânico-estatístico, caracterizado pela adoção de determinados instrumentos heurísticos específicos. Desta forma, Boltzmann estendeu e transformou o aparato conceitual e heurístico desenvolvido por seus predecessores em teoria cinética dos gases, contribuindo assim, de forma significativa na implantação de um programa mecânico-estatístico.

Fazendo uso da função de distribuição de velocidades moleculares de Maxwell foi possível à Boltzmann construir, no interior do seu programa de pesquisa, a transição de uma "abordagem cinética", de caráter mais propriamente mecânico e que caracterizou suas primeiras tentativas de resolução do problema da irreversibilidade termodinâmica, para uma "abordagem estatística". Esta última caracterizada basicamente pela utilização de novos elementos heurísticos tais como a noção de "ensemble estatístico" e o conceito de "probabilidade de estado".

\section{Referências}

[1] Cássio Costa Laranjeiras, O Programa de Pesquisa de Ludwig Boltzmann para a Mecânica Estatística: Uma Reconstrução Racional. Tese de Doutorado, Universidade de São Paulo, 2002.
[2] L. Boltzmann, (1866) in F. Hasenöhrl, editor, Wissenschaftliche Abhandlungen von Ludwig Boltzmann (Chelsea Publishing Company, Nova York, 1968).

[3] L. Boltzmann, (1868) in F. Hasenöhrl, editor, Wissenschaftliche Abhandlungen von Ludwig Boltzmann (Chelsea Publishing Company, New York, 1968).

[4] L. Boltzmann, (1871a) in F. Hasenöhrl, editor, Wissenschaftliche Abhandlungen von Ludwig Boltzmann (Chelsea Publishing Company, New York, 1968).

[5] L. Boltzmann, (1871b) in F. Hasenöhrl, editor, Wissenschaftliche Abhandlungen von Ludwig Boltzmann (Chelsea Publishing Company, New York, 1968).

[6] L. Boltzmann, (1871c) in F. Hasenöhrl, editor, Wissenschaftliche Abhandlungen von Ludwig Boltzmann (Chelsea Publishing Company, New York, 1968).

[7] L. Boltzmann, Mathematischnaturwissenschaftlichen Classe 90, 231 (1884). Citado por Renn em [Renn 2000].

[8] L. Boltzmann, (1885) in F. Hasenöhrl, editor, Wissenschaftliche Abhandlungen von Ludwig Boltzmann (Chelsea Publishing Company, New York, 1968).

[9] L. Boltzmann, (1896) Lectures on Gas Theory. (Dover Publications, New York 1995).

[10] S.G. Brush, Archive for History of Exact Sciences 4, 145 (1987).

[11] E.E. Daub, ISIS 60, 318 (1969).

[12] P.E.T. Ehrenfest, (1911). Begriffliche Grundlagen der statistischen Auffassung in der Mechanik., v. 4. Encyklopädie der mathematischen Wissenschaften, Teubner, Leipzig. Tradução Inglesa por M.J. Moravsik, The Conceptual Foundations of Statistical Mechanics (Cornell University Press, 1959, Dover Publications, 1990).

[13] G. Gallavotti, Journal of Statistical Physics 78, 1571 (1995).

[14] D.C.U. Garibaldi, Stud. Hist. Phil. Mod Phys. 28, 483 (1997).

[15] J.W. Gibbs, (1901). in A. Haas, editor, The Collected Works of J. Willard Gibbs (Longmans, Gren and Co., New York, 1931).

[16] M. Klein, in E.W. Thining, editor, The Boltzmann Equation of the Statistical Approach in Mechanics (Springer-Veríag, Cornel, 1973), p. 53-106.

[17] J.C. Maxwell, (1867), in Brush, S.G., editor, Kinetic Theory: The Nature of Gases and Heat (Oxford Pergamon Press, London, 1965).

[18] J. von Plato, Archive for History of Exact Sciences 42, 72 (1982).

[19] Y.M. Guttmann, The Concept of Probability in Statistical Physics. (Cambridge University Press, United Kingdom, 1999). 